\title{
Triage in the developing world-can it be done?
}

\author{
M A Robertson, E M Molyneux
}

\begin{abstract}
Aim-To assess guidelines for the emergency triage, assessment, and treatment (ETAT) of sick children presenting to hospitals in the developing world. This study pretested the guidelines in Malawi, assessing their performance when used by nurses compared to doctors trained in advanced paediatric life support (APLS). Methods-Triage was performed simultaneously by a nurse and assessing doctor on 2281 children presenting to the under $5 \mathrm{~s}$ clinic. Each patient was allocated one of three priorities, according to the ETAT guidelines. Any variation between nurse and assessor was recorded on the assessment forms.

Results-Nurses identified 92 children requiring emergency treatment and 661 with signs indicating a need for urgent medical assessment. One hundred and forty two $(6.2 \%)$ had different priorities allocated by the APLS trained doctor, but these children did not tend to need subsequent admission. Eighty five per cent of admissions were prioritised to an emergency or urgent category.

Conclusion-Although there are no gold standards for comparison the ETAT guidelines appear to reliably select out the majority of patients requiring admission. (Arch Dis Child 2001;85:208-213)
\end{abstract}

Keywords: triage; developing world; emergency care

Triage, that is the sorting of patients, has been used for some time in accident and emergency units in the developed world. Almost all that has been written about triage applies to well resourced departments in the western world. However, even well established triage systems such as those used in Australia, Canada, and the UK have not been extensively validated and the benefit of triage is still being argued. ${ }^{1-3}$

Blythin, ${ }^{4}$ in 1983 , suggested that the main objective of triage (at least in civilian practice) is immediate or early patient assessment to obviate an unscreened and possibly harmful delay. This interpretation applies across all types of environment and is particularly relevant to attempts of the World Health Organisation (WHO) to improve the management of sick children in a resource poor setting.

The WHO has developed guidelines for emergency triage, assessment, and treatment of sick children presenting to hospitals in the developing world (ETAT). ${ }^{5}$ Studies to pretest these guidelines have been done in $\mathrm{Brazil}^{6}$ and are now being performed in Malawi.

This validation study was conducted at the Queen Elizabeth Central Hospital (QECH) in
Blantyre. Circumstances differ in the two sites, malaria being absent in Brazil but endemic in Malawi. The primary objective set out by the WHO for the pretest study was to assess the performance of the draft WHO guidelines when used by nurses, compared to an advanced paediatric life support (APLS) ${ }^{7}$ trained doctor. Particular attention was to be given to the concordance of priority allocation and presence of priority signs.

\section{Methods}

STUDY SITE

The QECH in Blantyre is the teaching and referral hospital for the southern region of Malawi. It serves both adult and paediatric populations. The number of children seen per annum averages 100 000, about 10000 of who are admitted. Inpatient mortality is approximately $10 \%$.

Each weekday from 0730 to 1630 and every Saturday until 1300 the hospital holds an "under 5s clinic" (UFC) dedicated to the care of children with non-traumatic complaints. Outside these hours, all children are seen in the adult casualty department. At the UFC 200-700 children are treated daily. The department, when fully staffed has two clinical officers and four trained nurses. The nurses are responsible for registering and weighing children, as well as assisting in medical and surgical outpatient departments. They handle admission documentation in addition to their clinical responsibilities for patients. The department has a home craft worker (to supervise and advise parents) in the oral rehydration/ observation area and there is a hospital attendant.

Prior to the initiation of this project no formal triage occurred and most children were seen in order of presentation. Inevitably some of the sickest children died while awaiting assessment.

The ETAT guidelines use a system of priority signs (Appendix 1) to highlight children who require emergency treatment, urgent medical assessments, or those who may be put at risk by a prolonged wait.

\section{TRAINING}

The WHO provided a training plan that included manuals for trainers and trainees, and video and photographic material. We added role play, utilising locally available equipment (Appendix 2).

During the training period the nurses drew our attention to the significance of a child being very hot. They felt these children were at particular risk of having convulsions. This was not a priority sign in the initial WHO guidelines but we felt it warranted inclusion. 


\section{A B C $\mathrm{Cm} \mathrm{Cn} \mathrm{Cf} \mathrm{D} \mathrm{O}$}

P1 P2 P3

\author{
A = problem with airway \\ $\mathrm{B}=$ problem with breathing \\ $\mathrm{C}=$ problem with circulation \\ $\mathrm{Cm}=$ coma \\ Cn = convulsion \\ $\mathrm{Cf}=$ confusion \\ $\mathrm{D}=$ diarrhoea with dehydration \\ $\mathrm{O}=$ other priority sign \\ $\mathrm{P} 1=$ Emergency treatment required \\ P2 = Urgent assessment required \\ P3 = Ability to safely wait \\ Figure 1 Stamp for outpatient card.
}

The initial training at QECH was carried out in October 1997. It consisted of 10 two-hour sessions and was attended by eight registered nurses and four clinical officers. This included all nurses and two of the clinical officers working in UFC at the time of the study. There was a long time interval between the initial teaching and the study, so a refresher course was run in December 1998. This course comprised five two-hour sessions and all staff working in UFC during the study period attended, the majority of whom had been in the original training group.

Course students completed a written quiz at the end of training. A pass/fail was not given as all staff on the course improved. One trainee found dose calculation difficult and would have failed the quiz despite being an excellent traige assessment nurse. To avoid damage to staff morale we chose to give a certificate of attendance on completion of training, in preference to a certificate of success.

\section{STUDY PROTOCOL}

The study period was from December 1998 to January 1999. Study periods were limited to times when the UFC was fully staffed. Most assessments were done between 0830 and 1230 Monday to Thursday with the remainder done between 1330 and 1530 on the same days.

The study was carried out during the wet season, when the UFC is extremely busy, and there are many cases of malaria and malaria related illness. It was felt that using the guidelines in these conditions would test them in a "real life" situation.
Triage was performed by nurse and assessor using the guidelines shown in Appendix 1. Both assessors were APLS trained in the UK. Nurse triage was carried out on waiting children in order of their arrival and a priority was allocated to the patient. This was observed by one of the assessors, who recorded the nurses' observations on the assessment form followed by their own findings on the same form. Any difference in priority allocated by the assessor was documented.

Priority one (P1) was allocated to all children who were thought to need emergency treatment. Priority two (P2) was given to children who required an urgent assessment and priority three (P3) to those who could wait safely.

It was not possible to track the initial 700 children triaged through to final outcome because of lack of staff. The remaining 1581 cases were followed through to a decision to admit or discharge, with the clinical officer's admission diagnosis recorded. At the end of the study period the outcome of triaged children who had been admitted was determined as far as possible from available hospital records.

On completion of the formal study we tried to assess whether the nursing staff triaged as well when working unsupervised. We designed a stamp for the outpatient cards (fig 1).

On each of five consecutive mornings a triage nurse was allocated and patients were issued with stamped cards. The triage nurse was asked to assess each child according to the previously described guidelines. They then indicated on the card, by circling the appropriate letter, if the patient was an emergency (P1), priority (P2), or they could wait in the queue (P3). The nurse was also asked to indicate on the card in which systems they felt the abnormalities lay, for example, if in breathing, circle B. All children who were felt to require emergency treatments were transferred to the emergency room. Children thought to need priority assessments were taken to the front of the queue and all others were left to wait in order of presentation. The assessors were stationed at the clinical officers' desks and documented the priority assigned by the triage nurse, final outcome (admission, discharge), and admission diagnosis from the clinical officers.

\section{Results}

Altogether 2281 children were triaged during the study period and 1581 of these were followed through to admission or discharge. For all children admitted final outcome was sought from inpatient records. Thirty nine per cent of children (884) were aged 1 year or younger. Forty six per cent (1042) were over 1 year but less than 5 years, and the remaining 15 per cent (350) were 5 years and over.

The majority $(74 \%)$ of triage was done in the mornings. Nurses using the ETAT triage guidelines identified 92 patients requiring emergency treatment (P1) and 661 with priority signs (P2). Figure 2 shows the difference in priority allocation between the triage nurse and the assessing doctor. A total of 142 patients 


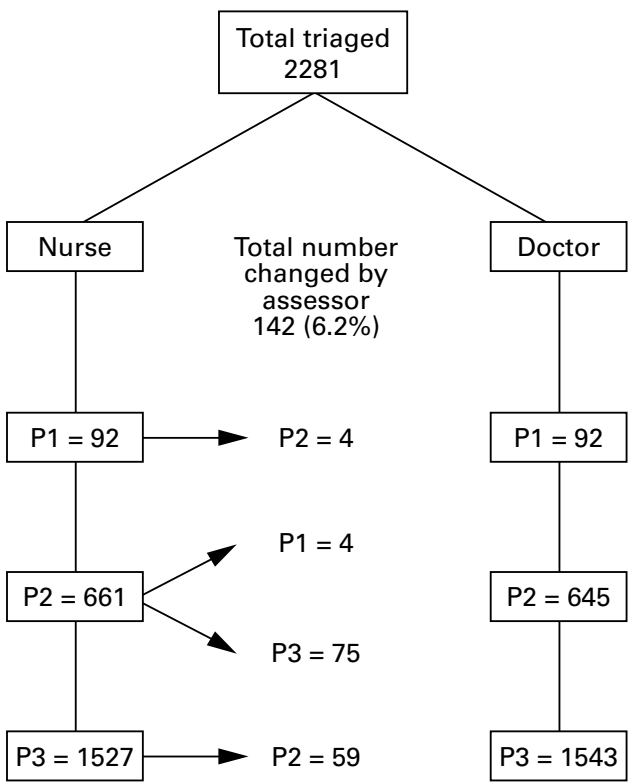

Figure 2 Change in nurse allocated triage category following assessment by doctor.

( $6.2 \%$ of those triaged) had priorities reassigned by the assessor. Four were downgraded from P1 to P2, one of whom was admitted. Four patients were moved from $\mathrm{P} 2$ to $\mathrm{P} 1$, three of whom were admitted and one discharged. Seventy five patients were reassigned from P2 to P3, only one of whom was thought to require admission; 59 changed from $\mathrm{P} 3$ to $\mathrm{P} 2$, of whom eight were admitted.

Table 1 shows the agreement between triage nurse and assessor on the presence or absence of priority signs. Some signs show a much larger variation than others, but all participants learned to assess well. One of the nurses found dose calculation in the emergency treatment difficult.

Of the 1581 children triaged with follow up, 236 were admitted. Ninety per cent of P1 were admitted, $32 \%$ of the P2, and only $3.5 \%$ of the P3 patients. Altogether $85 \%$ of admissions had been prioritised to emergency or urgent categories. Thirty one children died, four while awaiting triage and 27 after admission.

During the follow up period to assess the nurses' performance when working without the supervision of an assessor, 1000 children were triaged. This assessment was carried out over five sessions; table 2 outlines the results.

Sixteen children were allocated to $\mathrm{P} 1$, of whom $12(75 \%)$ were admitted; 335 were felt to be $\mathrm{P} 2$, of whom 124 (37\%) were admitted; and 649 were designated P3, of whom 17 $(2.5 \%)$ were admitted.

During the study period health centre referrals did not alter in type or number, as most referrals were appropriate. It was not possible to assess whether self referrals increased with the introduction of the triage system. Mothers appeared to understand that the sickest children were being seen in advance of those who were well, and sometimes presented directly to the triage nurse if their child was in extremis.

Between $80 \%$ and $96 \%$ of patients designated P1 or P2 by the nurses during nonsupervised triage were admitted. The exception is the triage conducted on the afternoon of day 4. On this afternoon there was no one available for dedicated triage duties, so the children were assessed by a trained nurse while being weighed. During this period the percentage of admissions prioritised by the nurse was only $50 \%$.

\section{Discussion}

The study was performed to assess the possibility of training nurses and paramedical workers to triage sick children and institute life saving treatments in a developing world setting. There were large variations in allocation of emergency and priority signs. For emergency signs assessors found double the number of children with an increased capillary refill, and assessed $50 \%$ more to be cold or lethargic than the nurses did. The largest difference in allocation of priority signs was for pallor, respiratory rate, and wasting.

Of the 2281 children triaged by the nursing staff using the ETAT guidelines 142 (6\%) had a different category allocated by the assessor. The category allocated seemed to correlate well with the likelihood of admission.

During the validation study in $\mathrm{Brazil}^{6}$ each patient was assessed by a nurse using the ETAT guidelines and then a paediatrician using APLS guidelines. This involved auscultation of the chest and recordings of pulse, respiratory rate, and heart rate. This was not a viable option in

Table 1 Priority signs identified by nurses compared to assessor

\begin{tabular}{|c|c|c|c|c|c|}
\hline Signs & Total agreed & Total nurse & Total doctor & $\begin{array}{l}\text { Nurse +ve } \\
\text { Doctor -ve }\end{array}$ & $\begin{array}{l}\text { Doctor +ve } \\
\text { Nurse -ve }\end{array}$ \\
\hline Respiratory distress & 42 & 53 & 47 & 11 & 5 \\
\hline Respiratory rate & 119 & 144 & 272 & 25 & 73 \\
\hline Hot & 194 & 218 & 211 & 24 & 17 \\
\hline Cold & 26 & 31 & 39 & 5 & 13 \\
\hline Capillary refill & 6 & 7 & 11 & 1 & 5 \\
\hline Lethargy & 267 & 287 & 402 & 20 & 135 \\
\hline Fits & 19 & 17 & 20 & 2 & 1 \\
\hline Diarrhoea & 115 & 136 & 123 & 21 & 8 \\
\hline Sunken eyes & 62 & 65 & 83 & 3 & 21 \\
\hline Skin pinch & 50 & 52 & 59 & 2 & 9 \\
\hline Oedema & 72 & 80 & 94 & 8 & 22 \\
\hline Wasting & 75 & 82 & 126 & 7 & 51 \\
\hline Pallor & 86 & 108 & 145 & 22 & 59 \\
\hline Tiny & 103 & 109 & 114 & 6 & 11 \\
\hline Irritable & 14 & 15 & 35 & 1 & 21 \\
\hline Referred & 53 & 55 & 55 & 2 & 2 \\
\hline
\end{tabular}


Table 2 Unsupervised triage: priorities and admissions of prioritised patients

\begin{tabular}{|c|c|c|c|c|c|c|}
\hline & Morning 1 & Morning 2 & Morning 3 & Morning 4 & Afternoon $4^{\star}$ & Morning 5 \\
\hline P1 & 0 & 2 & 0 & 8 & 1 & 6 \\
\hline Admitted & & 0 & & $7(88 \%)$ & $1(100 \%)$ & $5(83 \%)$ \\
\hline Discharged & & $2(100 \%)$ & & $1(12 \%)$ & 0 & $1(17 \%)$ \\
\hline P2 & 32 & 49 & 87 & 89 & 21 & 78 \\
\hline Admitted & $23(72 \%)$ & $27(53 \%)$ & $30(35 \%)$ & $28(31 \%)$ & $16(76 \%)$ & $16(20.5 \%)$ \\
\hline Discharged & $9(21 \%)$ & $22(45 \%)$ & $57(65 \%)$ & $61(68 \%)$ & $5(24 \%)$ & $62(79.5 \%)$ \\
\hline P3 & 92 & 169 & 95 & 111 & 47 & 182 \\
\hline Admitted & $6(6.5 \%)$ & $1(0.5 \%)$ & $5(5 \%)$ & $2(2 \%)$ & $7(15 \%)$ & $3(1.5 \%)$ \\
\hline Discharged & $86(93.5 \%)$ & $168(99.5 \%)$ & $90(95 \%)$ & $109(98 \%)$ & $40(85 \%)$ & $179(98.5 \%)$ \\
\hline $\begin{array}{l}\% \text { admissions } \\
\text { prioritised at triage }\end{array}$ & $80 \%$ & $96 \%$ & $86 \%$ & $94 \%$ & $50 \%$ & $87 \%$ \\
\hline
\end{tabular}

^No allocated nurse for triage.

the Malawi arm of the study, because of the environmental limitations of space, noise levels, and large numbers of waiting patients. Therefore both nurse and assessor used the ETAT guidelines.

The two assessors in this study, although both APLS trained, came from different backgrounds, one from accident and emergency and one from paediatrics. Neither had previous experience of working in the tropics. The assessments were not blinded. The assessors were aware of nurses' allocation of priority before they recorded their own. Nurses were informed if the assessors had altered the priority, as this affected the child's placement in the department. This in itself may have affected the nurses' next few assessments.

Our study revealed large variations in the number of priority and emergency signs assessed by nurses compared to the APLS trained doctors. The correlations did not change with time but trainees screened more rapidly with practice. These variations were also much larger than those seen in the previously published study from Brazil. ${ }^{6}$ There are several possible explanations. In the Brazilian study both paediatricians responsible for assessments were from the hospital in which the trial was being run. In the Malawi arm of the study both assessors came from the UK. The variation in assessment of pallor and wasting may be caused by differences in perceived or accepted norms between western and developing countries.

Lethargy is a subjective sign, and is open to different interpretation by individuals. There is also the possibility of cultural differences and expectations regarding childhood behaviour influencing assessment.

By contrast, increased respiratory rate is an objective sign. No numerical limits had been set to define increased respiratory rate, making it impossible to ascertain the true number wrongly assessed. Much has been written about the value of respiratory rate in the diagnosis of respiratory illness in children, ${ }^{8-16}$ and its lack of predictive value for hypoxaemia in a malaria endemic area ${ }^{17}$ There has also been considerable discussion about age specific normal values, ${ }^{18}$ the problems of accurate measurement, ${ }^{19-22}$ and its variability with fever. ${ }^{23}{ }^{24}$ However, in this study increased respiratory rate was used as an indicator of priority rather than specific pathology. In view of the confounding factors discussed above we have not described the variations as false negatives or positives, but as differences.

The accepted gold standard for assessment of core temperature is oral or rectal thermometry. However, actual values used to define fever are often selected at random. ${ }^{25}$ In UFC the facility for rectal measurement of temperature was not available and so fever was assessed by touch. This method has been shown to have a high negative predictive value, ${ }^{26}{ }^{27}$ and one study reported a $98 \%$ sensitivity for temperatures above $38.9^{\circ} \mathrm{C}$ in children under 2 years. ${ }^{28}$

It is interesting to see that the large variations in assessment of the presence of abnormal signs were not reflected in the allocation of priority categories. This may be a reflection of the experience of the nursing staff. Cioffi ${ }^{29}$ showed that triage was more likely to be correct if performed by more experienced nursing staff. The main difference was increased reference to previous patient encounters by the more experienced nurses.

It may also be that nursing staff allocated the correct priority to patients on the basis of a single sign, failing to pick up other indicators of priority. Does this mean that nurses are allocating priority on a quick eyeball assessment? The data collected at the follow up study of unsupervised triage would suggest not. During formal triage with a dedicated nurse, results were similar to the original study.

However, on the afternoon that patients were triaged while being weighed (eyeballed), the percentage of patients admitted who were prioritised fell from $86 \%$ to $50 \%$. An unpublished study (unpublished report 1997, Dr J Robson, consultant in paediatric A\&E, V Lavy, general practitioner) carried out in the same department prior to triage training, showed that of 50 severely ill children requiring admission only 13 were recognised by nursing staff.

Little has been written about inter- and intraobserver variation in triage. One study in the USA, ${ }^{30}$ looking at five scenarios, showed inter-rater agreement to be poor (kappa value $=0.347$ overall), and only $24 \%$ of participants rated the five cases the same severity in both phases. This lack of agreement in expected variations, and the lack of blinding of our assessors, makes it difficult to comment on the differences in category allocation in our study.

Correlation of triage category with likelihood of admission was good. Eighty three per cent of 
patients prioritised by the nurses during triage were admitted, and in the unsupervised triage this varied between $80 \%$ and $96 \%$ (excluding the afternoon of no formal triage). Admission rates for individual categories are comparable to those quoted for a recent implementation study of a new five level triage system in Boston. ${ }^{31}$

For 15 of the children who were triaged no record of their outcome was found. Tracing patients is a constant challenge because of the shortage of paper with overwriting on many records, and the lack of consistency in recording patients' details. Sometimes the child's date of birth is unknown, even to the mother, and many children pass through the department with the same, or similar names. We are, however, fairly confident of our mortality figures, as fatalities are presented each morning at a departmental meeting and these records are kept separately. The lack of such patient data also makes it impossible to know whether

EMERGENCY SIGNS patients re-presented or died after discharge. Because of the absence of reliable prestudy data there is no evidence for improvement in mortality and morbidity in UFC during the study period.

As systems presently in use in the developed world have not been extensively validated there is no gold standard to compare with the performance of the ETAT guidelines. However, use of the guidelines appears to reliably select out patients needing admission. This applies to use by both nurses and APLS trained doctors, and is independent of concordance of priority signs.

We would like to thank the Department of Child and Adolescent Health and Development (World Health Organisation) for supporting this study: Dr Vicky Lavy and Susan Champion for help in training, and testing the use of ETAT, and all the UFC staff who learned and practised triage with enthusiasm and skill.

\section{Appendix 1: Emergency signs}

Figure A1 presents the system of priority signs.

\section{EMERGENCY TREATMENTS}

Any sign positive

$\frac{\text { Airway and }}{\text { breathing }}$ A \& B $A \& B$

Circulation

$\mathrm{C}$

Not breathing Central cyanosis Severe respiratory distress

Obstructed breathing

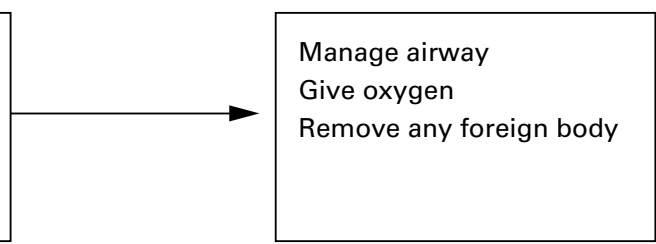

Cold hands Capillary refill over 3 seconds Weak fast pulse

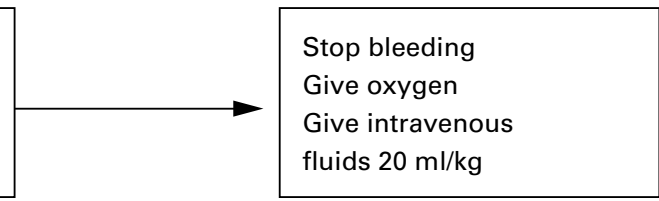

Coma

Convulsions Confusion $\mathrm{Cm} \mathrm{CnCf}$

Dehydration (child has vomiting or diarrhoea)

D

Unconscious
Convulsing now
Low blood sugar:
less than 2

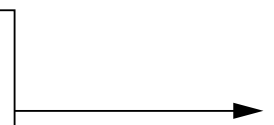

Manage airway

Give oxygen

Rectal diazepam

Give i.v. dextrose $10 \%$

Position child

Lethargy
Sunken eyes
Skin pinch more
than 3 seconds
fluids
Give i.v. or nasogastric

\section{PRIORITY SIGNS}

Severe wasting

Child under 2 months of age

Irritable or restless

Pallor

Major burn
Oedema both feet

Lethargy

Any respiratory distress

Urgent referral from another health facility

A child with any priority sign needs urgent assessment

Figure A1 Emergency signs. 


\section{Appendix 2: ETAT triage training}

A PILOT PROGRAMME

Duration: 10 two-hourly afternoon sessions.

Each day started with drills in the overall flow chart (Appendix 1).

- Day 1-Introduction. Purpose of triage. Flow chart of assessment for emergency and priority signs

- Day 2-Recognising clinical signs: examples, videos

- Day 3-Practice in identifying clinical signs: on paediatric ward; in UFC

- Day 4-Addition of emergency treatments to assessment algorithm

- Day 5-Exercises in rapid assessment: oral drills; with children in UFC

- Day 6-Airway and breathing management and practice. Oxygen delivery and airway scenarios.

- Day 7-Intravenous access and intraosseous infusion. Video examples. Intraosseous insertion practice on chicken legs.

- Day 8-Fluids in shock. Glucose administration. Scenarios.

- Day 9-Calculations for fluid and glucose requirements and diazepam dosage. Rectal administration of anticonvulsant drugs.

- Day 10-Severe dehydration. Management in well and malnourished children

- Written quiz-Wall charts and videos were supplied by WHO.

1 George S, Read S, Westlake L, et al. Evaluation of nurse triage in a British accident and emergency department. BMI 1992;304:876-8.

2 George S, Read S, Westlake L, et al. Nurse triage in theory and practice. Arch Emerg Med 1993;10:220-8.

3 Cooke MW, Jinks S. Does the Manchester triage system detect the critically ill? $\mathcal{F}$ Accid Emerg Med 1999;16:179-81. detect the critically ill? F Accid Emerg Med 1999;16:179-81. Blythin P. Would you

Mirror 1983;3:36-7.
Gove S, Tamburlini G, Molyneux E, et al (for the WHO

5 Gove S, Tamburlini G, Molyneux E, et al (for the WHO IMIC Referral Care Project). The development and techniassessment and treatment in developing countries. Arch Dis Child 1999;81:473-7.

6 Tamburlini G, Di Mario S, Maggi RS, et al. Evaluation of guidelines for emergency triage assessment and treatment in developing countries. Arch Dis Child 1999;81:478-82.

7 Advanced Life Support Group. Advanced paediatric life support - the practical approach, 2nd ed. London: BMJ Publishing Group, 1997

8 Cherian T, John TJ, Simoes E, et al. Evaluation of simple clinical signs for the diagnosis of acute lower respiratory clinical signs for the diagnosis of acut

9 Campbell H, Byass P, Lamont AC, et al. Assessment of clinical criteria for identification of severe acute lower resclinical criteria for identification of severe acute lower res-
piratory tract infection in children. Lancet 1989;ii:297-9.
10 Yaohua D, Foy HM, Zhu Z, et al. Respiratory rate and signs in roentgenographically confirmed pneumonia among children in China. Paediatr Infect Dis $\mathcal{F}$ 1995;14:48-50.

11 Berman S, Simoes E, Lanata C. Respiratory rate and pneumonia in infancy. Arch Dis Child 1991;66:81-3.

12 Morley CJ, Thornton AJ, Fowler MA, et al. Respiratory rate and severity of illness in babies under 6 months old. Arch Dis Child 1990;65:834-7.

13 Morley CJ. Respiratory rate and pneumonia in infancy [letter]. Arch Dis Child 1991;66:1001-4.

14 Onyongo FE, Steinhoff MC, Wafula EM, et al. Hypoxaemia in young Kenyan children with acute lower respiratory infection. BMF 1993;306:612-15.

15 Lucero MG, Tupasi TE, Gomez ML, et al. Respiratory rate greater than 50 per minute as a clinical indicator of pneumonia in Filipino children with cough. Rev Infect Dis 1990; 12(suppl 8):S1081-3.

16 Gove S, Kumar V. Simple signs and acute respiratory infections. Lancet 1988;ii:626-7.

17 O'Dempsey TJ, Todo JE. Chest infections in African children. Respiratory rate poor predictor of hypoxaemia [letter]. BMF 1993;306:1342.

18 Rusconi F, Castagneto M, Gagliardi L, et al. Reference values for respiratory rate in the first 3 years of life. Paediatrics 1994;94:350-5.

19 Simoes EAF, Roark R, Berman S, et al. Respiratory rate: measurement of variability over time and accuracy at different counting periods. Arch Dis Child 1991;66:1199203.

20 Gadomski AM, Khallaf N, El Ansary S, Black RE. Assessment of respiratory rate and chest indrawing in chil-
dren with ARI by primary care physicians in Egypt. Bull dren with ARI by primary care
World Health Org 1993;71:523-7.

$21 \mathrm{John}$ TJ, Cherian T. Determining the respiratory rate in children [letter]. World Health Forum 1991;12:74-5.

22 Gagliardi L, Rusconi F. Respiratory rate and body mass in the first three years of life. Arch Dis Child 1997;76:151-4.

23 O'Dempsey TJ, Lawrence BE, McArdle TF, et al. The effect of temperature reduction on respiratory rate in febrile illness. Arch Dis Child 1993;68:492-5.

24 Campbell H, Byass P, O'Dempsey TJ. Effects of body temperature on respiratory rate in young children [letter]. Arch Dis Child 1992;67:664.

25 Bonadio WA. Defining fever and other aspects of body temperature in infants and children. Pediatr Ann 1993;22:467$8,470-3$.

26 Bergeson P, Steinfeld H. How dependable is palpation as a screening method for fever? Clin Pediatr (Phila) 1974;13: 350-7.

27 Kresh M. Axillary temperature as a screening test for fever in children. F Pediatr 1984;104:596-9.

28 Banco L, Veltri D. Ability of mothers to subjectively assess the presence of fever in their children. Am 7 Dis Child 1984;138:976-9.

29 Cioffi J. Decision making by emergency nurses in triage assessments. Accid Emerg Nurs 1998;6:184-91.

30 Wuerz R, Fernandez CM, Alarcon J. Inconsistency of emergency department triage. Ann Emerg Med 1998;32:431-

31 Wuerz RC, Travers D, Ramine HY. Implementation of fivelevel triage at two university hospitals. Acad Emerg Med 2000;7:522. 